\title{
Sources of evidence in HIVIAIDS care: pilot study comparing family physicians and AIDS service organization staff Kasia E Stefanski ${ }^{1}$, C Shawn Tracy ${ }^{1}$ and Ross EG Upshur*1,2,3
}

Address: ${ }^{1}$ Primary Care Research Unit, Sunnybrook and Women's College Health Sciences Centre, 2075 Bayview Avenue, Room E3-49, Toronto, ON M4N 3M5 Canada, ${ }^{2}$ Department of Family and Community Medicine, University of Toronto, 257 McCaul Street, 2nd Floor, Toronto, ON M5T 2W5 Canada and ${ }^{3}$ Department of Public Health Sciences, University of Toronto, McMurrich Building, 12 Queen's Park Crescent West, Toronto, ON M5S 1A8 Canada

Email: Kasia E Stefanski - k.stefanski@utoronto.ca; C Shawn Tracy - shawn.tracy@sw.ca; Ross EG Upshur* - rupshur@idirect.com

* Corresponding author

Published: 09 July 2004

BMC Health Services Research 2004, 4:18 doi:10.1186/1472-6963-4-18

This article is available from: http://www.biomedcentral.com/l472-6963/4//8
Received: 12 February 2004

Accepted: 09 July 2004

(C) 2004 Stefanski et al; licensee BioMed Central Ltd. This is an Open Access article: verbatim copying and redistribution of this article are permitted in all media for any purpose, provided this notice is preserved along with the article's original URL.

\begin{abstract}
Background: The improvement of the quality of the evidence used in treatment decision-making is especially important in the case of patients with complicated disease processes such as HIVIAIDS for which multiple treatment strategies exist with conflicting reports of efficacy. Little is known about the perceptions of distinct groups of health care workers regarding various sources of evidence and how these influence the clinical decision-making process. Our objective was to investigate how two groups of treatment information providers for people living with HIVIAIDS perceive the importance of various sources of treatment information.
\end{abstract}

Methods: Surveys were distributed to staff at two local AIDS service organizations and to family physicians at three community health centres treating people living with HIVIAIDS. Participants were asked to rate the importance of 10 different sources of evidence for HIV/AIDS treatment information on a 5-point Likert-type scale. Mean rating scores and relative rankings were compared.

Results: Findings suggest that a discordance exists between the two health information provider groups in terms of their perceptions of the various sources of evidence. Furthermore, AIDS service organization staff ranked health care professionals as the most important source of information whereas physicians deemed AIDS service organizations to be relatively unimportant. The two groups appear to share a common mistrust for information from pharmaceutical industries.

Conclusions: Discordance exists between medical "experts" from different backgrounds relating to their perceptions of evidence. Further investigation is warranted in order to reveal any effects on the quality of treatment information and implications in the decision-making process. Possible effects on collaboration and working relationships also warrant further exploration.

\section{Background}

Evidence-based approaches to medical decision-making are assuming increased significance in health care. To be sure, there is greater focus on the incorporation of evidence derived from clinical research into clinical practice.
Randomized control trials are considered the gold standard of evidence and there is a tendency to give other forms of evidence - clinical expertise, intuition, qualitative data - a relatively lower profile [1]. Given the distinct nature of every patient/provider encounter, the unique context of 
individual patients, the distinguishing values and experiences of different physicians, and the breathtaking heterogeneity of modern health care, the assumption that it is possible to attain, or maintain, a "univocal notion of evidence" may be doubtful [2].

The definition of evidence is transitory; that is, it depends partly on current prevailing values in society that influence whether or not a particular practice is deemed successful, as well as the relative importance of specific outcome measures. Social constructs such as these vary by place and time [3]. There is a justifiable concern over what counts as evidence in the first place and how evidence is characterized, leading to a potential discordance between health information providers with respect to their understanding of the "best" evidence.

An exponential increase in the amount of available medical knowledge in the last few decades has hastened the desire to improve the quality of the evidence used in the medical decision-making process, indeed in all facets of health care. This is especially important in the case of patients with complicated disease processes such as HIV/ AIDS for which there is no known cure and for which multiple treatment strategies exist with conflicting reports of efficacy. The on-going shift towards evidence-based approaches in the education and treatment decision-making process of people living with HIV/AIDS (PHAs) can be seen as an attempt to link and introduce a new language in the diverse health care disciplines [2] and to make the decision-making process increasingly efficient and beneficial. Given the variety of sources of information and types of interventions that are potentially available, PHAs are especially vulnerable to their information providers.

It is thus important to understand how various medical information providers perceive different sources of evidence for treatment information in the context of their work and how this relates to differences in their backgrounds. The focus of this study was on two distinct groups of health information providers from distinctly different social and educational backgrounds, namely family physicians and AIDS service organization staff. The objective was to compare and contrast how these two professional groups perceive the importance of various sources of treatment information for HIV/AIDS care.

\section{Methods}

\section{Participants and setting}

Individuals eligible to take part in the study were treatment information providers for people living with HIV/ AIDS in the downtown urban core of Toronto, Canada. Participants were recruited at two specific types of health centres, namely community health centres (CHCs) that treat PHAs, and AIDS service organizations (ASOs) known to function as a resource for PHAs. The target sample comprised the following: (i) all 25 family physicians in active practice at three participating CHCs, and (ii) all 30 staff members employed at two participating ASOs. The latter group included community members (with and without formal credentials) as well as trained professionals with a background in health care and scientific research. The study received ethics approval from the Office of Research Services at the University of Toronto.

\section{Instrumentation}

The survey instrument was developed to quantify the perceived importance of various sources of evidence that can be used when making HIV/AIDS treatment decisions. Specifically, respondents were asked: "Below is a list of different sources that may contain information about HIV/ AIDS treatment. For each item, please circle the one number that best represents your opinion with respect to the importance of each of these different sources." Using a 5-point Likert-type scale ranging from "Very Important" to "Not At All Important," respondents rated the importance of the following 10 sources of treatment information: personal stories from people living with HIV/AIDS; news stories (articles in newspapers, magazines, etc.); word of mouth/information from peers; medical journals and textbooks; information from health care professionals; information from drug and pharmaceutical companies; Internet searches; clinical practice guidelines; information from AIDS service organizations; and conferences and presentations. Higher scores indicated greater perceived importance of the information source. The final section of the questionnaire requested typical demographic information, namely age, gender, and occupation. The survey instrument was developed with input from several health science researchers as well as staff at the Canadian AIDS Treatment Information Exchange (CATIE).

\section{Survey distribution}

In the spring of 2003, a total of 55 survey packages (questionnaires with informational cover letters) were distributed across the five participating health centres. One survey was distributed for each family physician employed at the three CHCs $(n=25)$ and one for each staff member at the two ASOs $(n=30)$. Participants were recruited by self-initiated pick-up of survey forms, which implied consent. Completed surveys were collected in sealed boxes at each location. No identifying information appeared on the surveys. The sites were visited several times during the following weeks to collect completed surveys.

\section{Data analysis}

Data were entered into a spreadsheet for statistical analysis in Microsoft Excel for Windows. Mean rating scores 
were calculated for family physicians and ASO staff on each of the 10 sources of evidence. Then, based on these mean scores, a crude relative ranking of sources (from most to least important) was produced for each of the two groups. Finally, we tested for statistically significant differences between the two groups using the Student's $t$-test. A separate analysis using non-parametric tests yielded similar results (not reported). Statistical significance was set at $\mathrm{p}<0.05$. Small cell sizes precluded statistical analysis of demographic differences between the two groups.

\section{Results}

In total, 23 of the 55 distributed surveys were completed and returned for an overall response rate of $42 \%$. The final achieved sample comprised 16 ASO staff and seven family physicians. Table 1 presents a demographic profile of the achieved sample. No demographic data were available for the target sample, thereby precluding a detailed comparison of respondents with non-respondents. When compared to available national physician workforce data [4], the achieved physician sample would appear to be broadly representative; however, the potential for response bias in our sample remains.

Using the $t$-test procedure, we tested for significant differences between the mean scores for family physicians and ASO staff. As indicated in Table 2, ASO staff attributed significantly more importance both to news stories and to ASOs than did the family physican group. Family physicians, on the other hand, rated the Internet and clinical practice guidelines significantly higher than did ASO staff. No statistically significant differences were observed for the six remaining sources of evidence.

Table 3 presents a comparison of the relative ranking of sources of treatment information for the two target groups. Family physicians ranked clinical practice guidelines as being most important, then medical journals and textbooks, followed by other health care professionals. In contrast, ASO staff ranked health care professionals, personal stories from PHAs, and ASOs as being the top three most important sources of information, respectively. Furthermore, it was also observed that each group ranked itself as being the third most important source; however, there was a striking difference in the way that each group ranked the other. While ASO staff ranked health care professionals as being the single most important source for treatment information, the physicians ranked ASOs in the eighth spot. Finally, pharmaceutical companies were judged by both groups to be a relatively unimportant source of information; indeed, this source was ranked $9^{\text {th }}$ and $10^{\text {th }}$ by physicians and ASO staff, respectively.

\section{Discussion}

The findings from this pilot project suggest that there is discordance between health information providers on the relative importance of different sources of evidence for treatment information. This study also demonstrated that the principal treatment information providers for PHAs, namely physicians and ASO staff, do not perceive each other as being equally credible information sources.

The present findings are believed to represent the first published data comparing physicians and ASO staff in terms of their preferences and attitudes towards different sources of evidence and the treatment decision-making process. The external validity of the study is limited by the small sample size and moderate response rate. Moreover, our achieved sample may not accurately represent the target populations, with regard to age and gender distribution. Also, the study sample was restricted to the urban core of a large metropolitan centre and participants were self-selected.

The present results indicate significant differences in how medical information providers from different backgrounds perceive the importance of various sources of treatment information for HIV/AIDS care. This is consistent with similar comparative studies that have focused on health care providers from various fields, including oncologists and naturopaths [5], nurses and physiotherapists [6], midwives and obstetricians [7], nurse practitioners and managers [8], full-time academic medical faculty and community-based volunteer medical faculty [9], as well as professionals in the health authorities and professionals providing primary care [10]. Novak and Chapman reported that oncologists and naturopaths differed in their advice concerning diet in breast cancer, citing different decision-making or knowledge-construction systems, based on the use of various sources of evidence, as the cause. Similarly, Stewart found that that the definition of evidence varies widely among health practitioners and contended that it may be framed or constrained by the culture of a particular health service.

Palfreyman and colleagues showed that despite the fact that both physiotherapists and nurses access a wide variety of sources of evidence these are not all necessarily being used equally as a basis for clinical practice. The study by Beasley and Woolley comparing the perception of the importance of evidence-based medicine (EBM) in the everyday practice of full-time medical faculty members vs community-based primary care and subspecialty faculty revealed that community-based faculty were less informed about EBM and used evidence-based sources of information less often than their full-time counterparts. Le May and associates concluded from their study that nurse practitioners differed from managers in their per- 
Table I: Demographic characteristics of achieved sample.* Values are numbers (percentages) unless otherwise indicated**

\begin{tabular}{|c|c|c|c|}
\hline Characteristic & Family physicians $(n=7)$ & ASO staff $(n=16)$ & Total $(\mathbf{N}=23)$ \\
\hline \multicolumn{4}{|l|}{ Age Group: } \\
\hline $18-30$ yrs & I (14) & $6(40)$ & $7(32)$ \\
\hline $31-40$ yrs & $4(57)$ & $7(47)$ & II (50) \\
\hline $4 I-50$ yrs & I (14) & $2(13)$ & $3(14)$ \\
\hline $5 \mathrm{I}-60 \mathrm{yrs}$ & $I(14)$ & $0(0)$ & I (5) \\
\hline \multicolumn{4}{|l|}{ Gender: } \\
\hline Male & $7(100)$ & $7(47)$ & $14(64)$ \\
\hline Female & $0(0)$ & $8(53)$ & $8(36)$ \\
\hline
\end{tabular}

* Some surveys were returned with missing data. ** Percentages may not sum up to 100 due to rounding.

Table 2: Mean importance ratings of various sources of evidence by physicians and ASO staff

\begin{tabular}{lccc}
\hline Source of evidence & Family physicians & ASO staff & P value of difference \\
\hline Personal stories from PHAs* & 3.71 & 4.38 & 0.08 \\
News stories & 2.29 & 3.40 & 0.01 \\
Word of mouth & 3.57 & 3.47 & 0.81 \\
Medical journals/books & 4.43 & 4.25 & 0.61 \\
Health care professionals & 4.29 & 4.40 & 0.74 \\
Pharmaceutical companies & 3.17 & 3.25 & 0.84 \\
Internet & 3.86 & 3.27 & 0.03 \\
Clinical practice guidelines & 4.67 & 3.87 & 0.03 \\
ASOs** & 3.43 & 4.31 & 0.03 \\
Conferences & 4.14 & 4.06 & 0.86 \\
\hline
\end{tabular}

* People living with HIVIAIDS ** AIDS service organization

Table 3: Relative ranking of the importance of various sources of HIVIAIDS treatment information by physicians and ASO* staff

\begin{tabular}{lll}
\hline Rank & Family physicians & ASO staff \\
\hline$\# \mathbf{1}$ & Clinical practice guidelines & Health care professionals \\
$\# \mathbf{2}$ & Medical journals/books & Personal stories from PHAs** \\
$\# 3$ & Health care professionals & ASOs* \\
$\# 4$ & Conferences & Medical journals/books \\
$\# 5$ & Internet & Conferences \\
$\# 6$ & Personal stories from PHAs** & Clinical practice guidelines \\
$\# \mathbf{7}$ & Word of mouth & Word of mouth \\
$\# 8$ & ASOs* & News stories \\
$\# 9$ & Pharmaceutical companies & Internet \\
$\# 10$ & News stories & Pharmaceutical companies
\end{tabular}

* AIDS service organization ** People living with HIVIAIDS

ceptions of the nature and role of research in practice. Finally, Coleman and Nicholl showed that professionals in the health authorities were much more likely to be aware of evidence-based guidance on health-care decisions and consulted more evidence-based sources than hospital consultants or primary care practitioners. Viewed collectively, these studies suggest that there is substantial variation in the knowledge, use, and perceived influence of research and different sources of evidence across health care fields. 
In the present study, the observed rankings of different information sources indicate that physicians gave most credence to those sources that were based on a solid scientific background and likely to be peer reviewed, as well as to individual sources with similar scientific and medical training to their own. In contrast, ASO staff gave relatively more importance to personal narratives and especially to experience developed through communication. The ASO group actually attributed greater importance to personal stories from clients than to their "own kind" (i.e., other ASOs). This pattern of findings suggests that, in terms of using information for a decision-making process, physicians may be more influenced by quantitative sources of evidence, whereas ASO staff may be more oriented to qualitative sources. The findings further suggest that the background of the experts may influence their preference and epistemological commitment to a particular type of evidence.

This study highlights how modern medical care is centred around two principal paradigms: "evidence-based medicine" and "patient-centred medicine [11-13]. In order to address this dichotomy, it is necessary to integrate various sources of evidence and accept their complementary roles in a symbiotic relationship. Bridging the gap will require unprecedented levels of collaboration between all groups participating in health care decisions $[14,15]$.

Despite the observed differences in the rankings of sources of evidence, it was observed that both groups appeared to share a common mistrust of information provided by drug companies. This is consistent with prior research indicating that health care workers are sceptical of the credibility of evidence obtained from the pharmaceutical industry [16]. Studies have indeed shown that research funded by drug companies is more likely to have outcomes favouring the sponsor [17].

The family physicians in this sample regard ASOs as a relatively unimportant source of HIV/AIDS treatment information, even less important than the Internet or word of mouth. Further study is warranted to investigate the causes and consequences of the professional respect and confidence (or lack thereof) of one group of health information providers towards another. The present results suggest that physicians and allied health care workers do not necessarily perceive each other as equally credible sources of evidence for treatment information. The implications of this finding need to be investigated in order to determine how this affects the collaboration between health care professionals. The existence of a stronger professional partnership between physicians and ASO staff could help increase the quality, efficacy, and trust in the treatment information provided.
Finally, investigating the existence of discordance of perceptions is essential for another reason: it could influence the quality of the education of PHAs, thus affecting a major determinant of their health. It would be of interest to determine how discordance affects the quality of the treatment information given to PHAs, as well as their satisfaction with the information dissemination process. This highlights the importance of designing better "measurement tools" that effectively assess health care provider attitudes toward and understanding of evidence. Indeed, there is a need to produce, package, and disseminate evidence in ways that better reflect provider preferences.

\section{Competing interests}

None declared.

\section{Authors' contributions}

KES initiated and designed the study, coordinated data collection, performed the statistical analysis, prepared the first draft of the manuscript, and will act as guarantor. CST participated in the analysis and interpretation of the data and contributed to the writing and editing of the manuscript. REGU consulted on the design of the study, assisted with data analysis and interpretation, and participated in writing the manuscript. All authors have read and approved the final version.

\section{Acknowledgements}

The authors would like to thank Dr. Bart Harvey of the Department of Family and Community Medicine, University of Toronto for his assistance with the statistical analysis. The "Determinants of Community Health" course in the Faculty of Medicine, University of Toronto supported the project and assisted with its initiation. The Canadian AIDS Treatment Information Exchange (CATIE) helped with survey design. We also gratefully acknowledge the physicians and ASO staff who voluntarily participated in our study. Thanks to Shari Gruman for editing and formatting the manuscript. Dr. Upshur is supported by a New Investigator Award from the Canadian Institutes of Health Research and a Research Scholar Award from the Department of Family and Community Medicine at the University of Toronto.

\section{References}

I. Tonelli MR: The limits of evidence-based medicine. Respir Care 200।, 46: |435-|440. discussion |440-|43|

2. Upshur RE: If not evidence, then what? Or does medicine really need a base? J Eval Clin Pract 2002, 8: I I3-I I9.

3. Leeder SR, Rychetnik L: Ethics and evidence-based medicine. Med J Aust 2001, I 75:16I-164.

4. College of Family Physicians of Canada: National Family Physician Workforce Survey (The Janus Project). Mississauga, Ontario: College of Family Physicians of Canada 200I.

5. Novak KL, Chapman GE: Oncologists' and naturopaths' nutrition beliefs and practices. Cancer Pract 200I, 9:|4|-|46.

6. Palfreyman S, Tod A, Doyle J: Comparing evidence-based practice of nurses and physiotherapists. Br J Nurs 2003, I 2:246-253.

7. Stewart M: Whose evidence counts? An exploration of health professionals' perceptions of evidence-based practice, focusing on the maternity services. Midwifery 200I, I 7:279-288.

8. Le May A, Mulhall A, Alexander C: Bridging the research-practice gap: exploring the research cultures of practitioners and managers. J Adv Nurs 1998, 28:428-437. 
9. Beasley BW, Woolley DC: Evidence-based medicine knowledge, attitudes, and skills of community faculty. J Gen Intern Med 2002, 17:632-639.

10. Coleman P, Nicholl J: Influence of evidence-based guidance on health policy and clinical practice in England. Qual Health Care 200I, 10:229-237.

II. Bensing J: Bridging the gap. The separate worlds of evidencebased medicine and patient-centered medicine. Patient Educ Couns 2000, 39: 17-25.

12. Krupat E, Rosenkranz SL, Yeager CM, Barnard K, Putnam SM, Inui TS: The practice orientations of physicians and patients: the effect of doctor-patient congruence on satisfaction. Patient Educ Couns 2000, 39:49-59.

13. Bensing JM, Verhaak PF, van Dulmen AM, Visser AP: Communication: the royal pathway to patient-centered medicine. Patient Educ Couns 2000, 39: I-3.

14. Jadad AR, Enkin MW: The new alchemy: transmuting information into knowledge in an electronic age. CMAJ 2000, 162: $1826-1828$

15. Upshur RE, VanDenKerkhof EG, Goel V: Meaning and measurement: an inclusive model of evidence in health care. J Eval Clin Pract 200I, 7:91-96.

16. Tracy CS, Dantas GC, Upshur RE: Evidence-based medicine in primary care: qualitative study of family physicians. BMC Fam Pract 2003, 4:6.

17. Lexchin J, Bero LA, Djulbegovic B, Clark O: Pharmaceutical industry sponsorship and research outcome and quality: systematic review. $B M J$ 2003, 326: I I67-II 170 .

\section{Pre-publication history}

The pre-publication history for this paper can be accessed here:

http://www.biomedcentral.com/1472-6963/4/18/prepub

Publish with Biomed Central and every scientist can read your work free of charge

"BioMed Central will be the most significant development for disseminating the results of biomedical research in our lifetime. "

Sir Paul Nurse, Cancer Research UK

Your research papers will be:

- available free of charge to the entire biomedical community

- peer reviewed and published immediately upon acceptance

- cited in PubMed and archived on PubMed Central

- yours - you keep the copyright 